\title{
APPLICATION OF QUASI-FORBIDDEN REFLECTIONS FOR DETERMINATION OF COMPOSITION OF PSEUDOBINARY SEMICONDUCTORS
}

\author{
J. Bąk-Misiuk, W. Paszkowicz and J. Domagala \\ Institute of Physics, Polish Academy of Sciences \\ Al. Lotników 32/46, 02-668 Warszawa, Poland
}

\begin{abstract}
An application of X-ray quasi-forbidden reflection method of composition determination for $\mathrm{A}^{\mathrm{II}} \mathrm{B}^{\mathrm{VI}}$ pseudobinary compounds is discussed. Three typical cases of the intensity dependence on the composition, as well as the choice of the most suitable reflection are presented.
\end{abstract}

PACS numbers: $61.10 . \mathrm{Wg}$

\section{Introduction}

Optical and electrical properties of ternary and quaternary compounds depend strongly on the crystal composition. Knowledge of the composition enables prediction of the mentioned above properties and evaluation of the energy gap. The aim of our paper is to extend the X-ray quasi-forbidden reflection method ("XFR") [1] for the composition determination of ternary II-VI compounds.

\section{Determination of ternary solid solution composition}

The diffracted intensity of $\mathrm{X}$-rays is determined by the structure factor $F$ :

$$
F=\sum_{j} f_{j} \exp \left(-M_{j}\right) \exp \left(2 \pi \mathrm{i} h \cdot r_{j}\right)
$$

where $f_{j}$ is an atomic scattering factor, $M_{j}$ is a temperature factor, $h$ is a vector of the Miller indices and $r_{j}$ is an atomic coordinate.

For sphalerite type structure the reflections satisfying the relation

$$
h+k+l=4 n+2 \quad(n=0,1,2 \ldots)
$$

have low intensity if the $f_{\mathrm{II}}$ and $f_{\mathrm{VI}}$ values are similar, because $F_{h k l}$ is proportional to $f_{\mathrm{II}}-f_{\mathrm{VI}}$. In general, for any structure type these allowed reflections for which $F_{h k l}$ is extremely low due to compensated $f$ values, are called "quasi-forbidden" ("QF"). 
For ternary solid solution $\mathrm{A}_{1-x}^{I I} \mathrm{~A}_{x}^{I I} \mathrm{~B}^{V I}$ and for $\mathrm{A}^{I I} \mathrm{~B}_{1-x}^{V I} \mathrm{~B}_{x}^{V I^{\prime}}$ the expression (1) is replaced by

$$
F=4\left[(1-x) f_{\mathrm{II}}+x f_{\mathrm{II}}-f_{\mathrm{VI}}\right]
$$

or

$$
F=4\left[f_{\mathrm{II}}-(1-x) f_{\mathrm{VI}}-x f_{\mathrm{VI}^{\prime}}\right] .
$$

Weak dependence of $F$ on temperature is neglected here. QF reflections are the most suitable for composition investigation of perfect, as well as distorted crystals, because in both cases the kinematical theory of X-ray diffraction may be applied for calculation of intensity $[2,3]$. Using of this theory yields the following expression for the ternary to binary intensity ratio [1]:

$$
\frac{I(x)}{I(0)}=\frac{|F(x)|^{2}}{|F(0)|^{2}} \frac{\mu(0) \sin 2 \theta_{0}\left(1+\cos ^{2} 2 \theta\right)}{\mu(x) \sin 2 \theta\left(1+\cos ^{2} 2 \theta_{0}\right)},
$$

where $I(0)$ and $I(x)$ denote the measured integrated intensity of the QF reflection for the binary and ternary compound, respectively, $\mu(x)$ is the linear absorption coefficient of the ternary compound, $\theta_{0}$ and $\theta$ are the Bragg angles for the QF reflection of the binary and ternary compound, respectively.

Equation (5) is valid for bulk crystals and for thin layers of thickness higher than the X-ray penetration depth. If these conditions are satisfied, the composition of the crystal may be directly derived from the experimentally determined value of $I(x) / I(0)$, using the dependence described by Eq. (5).

\section{Results}

The values of $I(x) / I(0)$ were calculated for $\mathrm{Cd}_{1-x} \mathrm{Mn}_{x} \mathrm{Te}, \mathrm{CdTe}_{1-x} \mathrm{Se}_{x}$ and $\mathrm{Zn}_{1-x} \mathrm{Hg}_{x}$ Te for $\mathrm{X}$-ray wavelength $\mathrm{Cu} K_{\alpha_{1}}$. The calculations were performed using the literature data for II-VI solid solutions lattice parameters for $\mathrm{CdTe}_{1-x} \mathrm{Se}_{x}$ and $\mathrm{Zn}_{1-x} \mathrm{Hg}_{x}$ Te based on linear extrapolation of CdTe, CdSe, HgTe, and ZnTe data (values from [4]) and for $\mathrm{Cd}_{1-x} \mathrm{Mn}_{x} \mathrm{Te}$ (from [5]). Polynomial expressions for the real and imaginary parts of the atomic scattering factor were applied [6]. There are three possible kinds of dependence of the QF intensity ratio on $x$. This dependence for the ternary compounds is classified according to the relation between the atomic scattering factors of the components.

In the considered examples $f^{0}$ denotes the real part of $f$ for $\sin \theta / \lambda=0.5$ corresponding approximately to the diffraction angle of 600 reflection using $\mathrm{Cu}$ $K_{\alpha}$ radiation.

Example 1: $\mathrm{Cd}_{1-x} \mathrm{Mn}_{x} \mathrm{Te}$

QF reflections exist for CdTe because $f_{\mathrm{Cd}}^{0}(24.4)$ and $f_{\mathrm{Te}}^{0}(26.9)$ are similar. Substituted atom $(\mathrm{Mn})$ - has a much different $f^{0}$ value $\left(f_{\mathrm{Mn}}^{0}=11.1\right)$, therefore the intensity of QF reflections (222), (420) and (600) increases with $x$ by 25-40 times across the solubility range in a similar way as for previously reported $\mathrm{Ga}_{1-x} \mathrm{Al}_{x} \mathrm{As}$ [1]. 

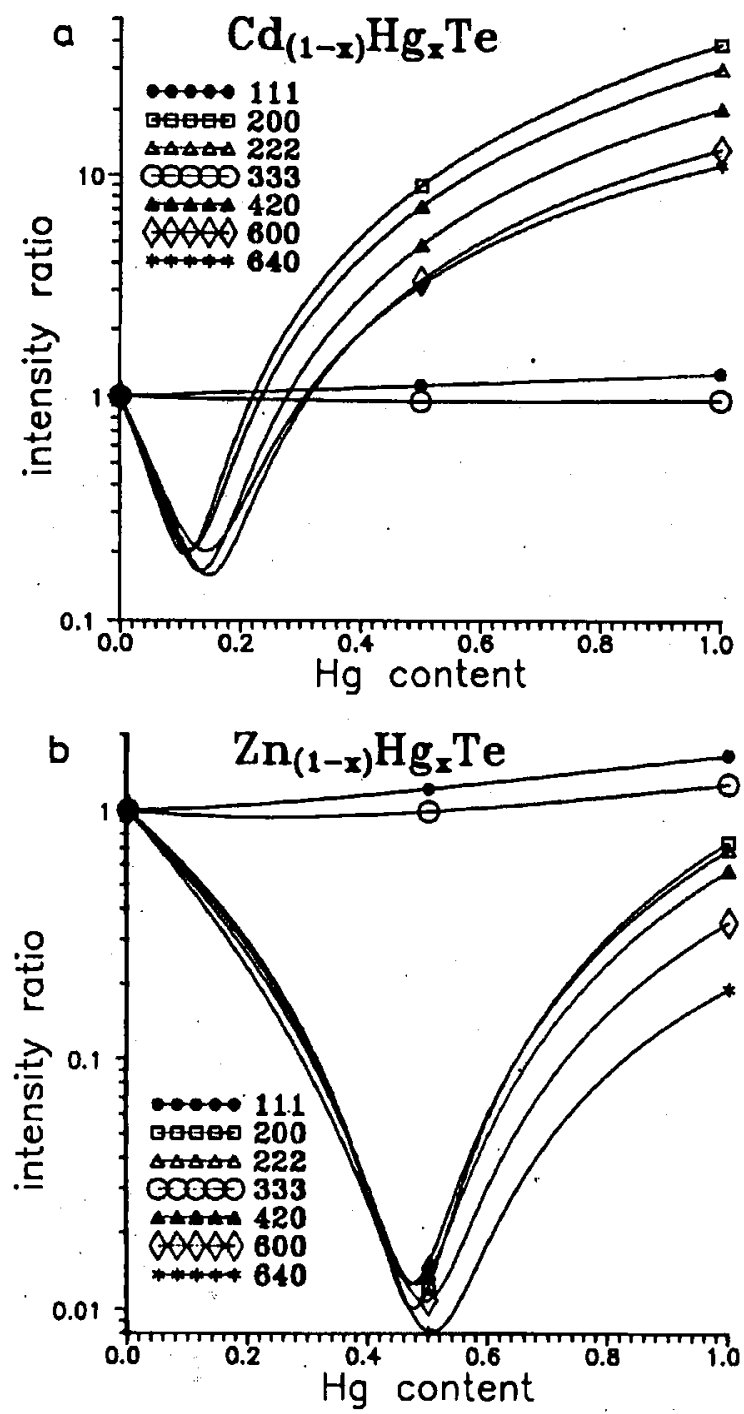

Fig. 1. Intensity ratio as a function of $\mathrm{Hg}$ content in $\mathrm{Cd}_{1-x} \mathrm{Hg}_{x} \mathrm{Te}$ (a) and $\mathrm{Hg}$ content in $\mathrm{Zn}_{1-x} \mathrm{Hg}_{x} \mathrm{Te}$ (b) for QF reflections 200, 222, 420,600 and 640 and for allowed 111 and 333 reflection.

Example 2: $\mathrm{Cd}_{1-x} \mathrm{Hg}_{x} \mathrm{Te}$

This example differs from the first one by the substituted atom. $f_{\text {Te }}^{0}(26.9)$ lies between $f_{\mathrm{Cd}}^{0}(24.4)$ and $f_{\mathrm{Hg}}^{0}(44.4)$. This causes that QF reflections for binary CdTe are weak in a very broad $x$ range, and for an intermediate composition 
(about $x=0.10-0.15$ ) the intensity ratio almost vanishes due to compensation of the atomic scattering factors in Eq. (3) (see Fig. 1a); it does not vanish completely due to the small imaginary part of the atomic scattering factor.

Example 3: $\mathrm{Zn}_{1-x} \mathrm{Hg}_{x} \mathrm{Te}$

The difference of $f$ values makes that reflections satisfying Eq. (2) are strong for binary $\mathrm{HgTe}$, as well as for $\mathrm{ZnTe}$. However, in this particular case $f_{\mathrm{Te}}^{0}(26.9)$ lies between $f_{\mathrm{Zn}}^{0}$ (13.9) and $f_{\mathrm{Hg}}^{0}$ (44.4). This causes that for an intermediate composition (about $x=0.5$ ) the intensity ratio almost vanishes as in the second example (see Fig. 1b). Therefore, the (200), (222), (420), (600), and (640) reflections become QF-type around this composition of the ternary compound.

Among the investigated QF reflections the (222), (420), and (600) are the most suitable for composition investigation because their intensity only weakly depends on the defect structure in the full solubility range.

For all considered examples the intensity ratio of allowed reflections 111 and 333 changes by only several percent in the whole solubility range.

\section{Discussion}

The QF reflections may be found only in a part of II-VI systems. The intensity of QF reflection is very sensitive to the composition. It changes 5 times in the $x$ range $[0,0.15]$ in $\mathrm{Cd}_{1-x} \mathrm{Hg}_{x} \mathrm{Te}$ or by 2 orders of magnitude in the whole composition range of all three systems. In addition, it is not much influenced by the defect structure of the crystal. The existence of the intensity-ratio minimum in a number of systems strongly increases the sensitivity of this ratio to composition. However, the composition value in some $x$-subrange becomes ambiguous. The ambiguity may be overcome by completing the intensity measurements with another suitable experimental method. Fujimoto [7] has mentioned the possibility of application of the XFR method to binary $\mathrm{ZnSe}$ and CdTe. The above considerations show that the class of materials which may be investigated by this method is wider and includes a number of solid solutions based on II-VI compounds.

\section{References}

[1] J. Bąk-Misiuk, W. Paszkowicz, J. Domagała, S. Miotkowska, Z.R. Żytkiewicz, J. Cryst. Growth 126, 168 (1993).

[2] H. Cole, N.R. Stemple, J. Appl. Phys. 33, 2227 (1962).

[3] A. Cockerton, G.S. Green, B.K. Tanner, Mater. Res. Soc. Symp. Proc. 138, 65 (1989).

[4] S. Ignatowicz, A. Kobendza, Semiconducting Thin Films of $A^{I I} B^{V I}$ Compounds, PWN, Warszawa 1990.

[5] J. Bąk, U. Dębska, R.R. Gałązka; G. Jasiołek, E. Mizera, in: Proc. XI Int. Crystallographic Congress Warsaw 1978, Ed. S. Olejnik, Institute for Low Temperature and Structure Research, p. 245.

[6] International Tables for $X$-ray Crystallography, Vol. IV, Kynoch Press, Birmingham 1974.

[7] İ. Fujimoto, Jpn. J. Appl. Phys. 23, L287 (1984). 